(Aus der geburtshülflich-gynäkologischen Klinik der Universität in Genua.)

\title{
Ueber die Widerstandskraft von Thieren während der Schwangerschaft und im Puerperium gegen Infection und Intoxication.
}

(Vorläufige Vittheilung zur Indication der Unterbrechung der Schwangerschaft wegen innerer Krankheiten.1)

$$
\text { Von }
$$

Prof. Dr. Bossi,

Director.

Durch den Wunsch, einen kleinen Beitrag zur Lehre des so wichtigen Problems der Indication zur Unterbrechung der Schwangerschaft wegen innerer Krankheiten zu geben, wurde ich veranlasst, die Widerstandskraft der Thiere während der Schwangerschaft und des Puerperiums gegen Infection und Intoxication zu prüfen. Zur Lösung dieser Frage giebt es kein rationelleres und wissenschaftlich positiveres Mittel, als experimentell die grössere oder geringere Widerstandskraft der Kreissenden gegen infectiöse Stoffe zu prüfen, da ja diese Stoffe (Tuberculose, Typhus, Pneumonie etc.) thatsächlich am häufigsten das pathogenetische Element der internen Complicationen während der Schwangerschaft bedingen und so auch die Hauptgrundlage der Indication zur Unterbrechung derselben wegen innerer Krankheiten abgeben.

Es wäre gewiss nicht richtig gewesen, Experimente bei einzelnen Thieren während der Schwangersehaft zu machen, ohne sie auch

1) Vorgetragen in der Discussion zum 1. Thema des IV. internationalen Congresses für Geburtshülfe und Gynäkologie. Rom. Septbr. 1902. 
auf das Puerperium auszudehnen, ihre Wirkung auf den Fötus zu untersuchen und auf das Säugungsgeschäft. Es ist mir nur leid, dass die zahlreichen Schwierigkeiten solcher Experimente nicht gestattet haben, sie zu vervollständigen. So muss ich das Folgende nur als vorläufige Mittheilung bieten.

Sowohl Pathologen als Hygieniker geben die erhöhte Disposition für Infectionen aus verschiedenen Einflüssen zu, welche den Organismus schwächen, sei es wegen übermässigen Kraftverbrauches, sei es wegen ungenügenden oder gestörten Stoffwechsels. So ist es von Canalis und Morpurgo für den Hunger bewiesen worden, von Henkins und Jener für Aenderung der gewohnten Ernährungsweise, von Pernice und Alessi für den Durst, von Rodet und Serafini für Blutverluste, von Gärtner und Bonome für Hydrämie, von Pasteur für Kälte, von Gibié für Hitze, von Bouchard und Charrin für Ueberanstrengung, von Fodor für Vergiftungen und von anderen für andere Ursachen, welche den menschlichen Organismus schwächen. Es ist deshalb gewiss interessant, solche Untersuchungen auch für die Schwangerschaft anzustellen, einen Zustand, in welchem der Organismus für den Stoffwechsel von zwei Existenzen aufzukommen hat, mit einer einzigen Bahn für die Ausscheidung von schädlichen und unbrauchbaren Stoffen, in welchem also die Organe höheren Leistungen ausgesetzt sind, das Gleichgewicht zu erhalten.

Von vorneherein entsteht die Vermuthung, dass in diesem, wenn auch rein physiologischen Zustande wegen der doppelten Arbeitsleistung die Widerstandskraft gegen infectiöse Stoffe herabgesetzt sei, oder wenigstens dass der Organismus während der Schwangerschaft sich im Kampfe um's Dasein in ungünstigeren Verhältnissen befinde als ausserhalb derselben. Doch ist nöthig, dies experimentell zu beweisen, was, wie ich glaube, bis jetzt noch nicht unternommen worden ist, wie viel Untersuchungen auch angestellt wurden, andere Nebenerscheinungen festzustellen (siehe das Literaturverzeichniss).

Um zur Lösung dieser Frage beizutragen, haben wir dies Jahr in unserer Anstalt eine Reihe von Thierexperimenten unternommen (an Kaninchen, Meerschweinchen bis jetzt 79) indem wir versuchten, die Widerstandskraft der schwangeren Thiere gegen die gewöhnlicheren Infectionsstoffe zu prüfen im Vergleich zu derjenigen nichtschwangerer Thiere der gleichen Gattung.

Die nämlichen Experimente wurden bei Gelegenheit auch an 
Thieren im Puerperium unternommen; dabei wurde niemals (wenn die Möglichkeit vorhanden war) versäumt, mit den zuverlässigsten Mitteln den Infectionsstoff im Product der Schwangerschaft zu suchen, um seinen Uebergang auf den Fötus zu constatiren, ebenso warde in der Milch nach dem eingeimpiten Mikroorganismus gefahndet.

Es warde alle Mühe darauf verwendet, die Schwierigkeiten, denen man bei solchen Untersuchungen begegnet, zu überwinden, um ein möglichst genaues Maass zur Beurtheilung zu bekommen. Es wurde daher auch jedesmal sorgfältig darauf geachtet, unter identischen Bedingungen zu experimentiren - Thierrace, Gescblecht, Gewicht. Bei letzterem wurde, wenn der Unterschied zu gross war, versucht ihn zu compensiren, indem man die Dosis des einzuimpfenden Stoffes vermehrte ader verminderte -, sodass man unter den gleichen Bedingungen und gleichzeitig bei Thieren im Zustande der Schwangerschaft, des Puerperium und im normalen Zustande beobachten konnte.

Die Versuche worden immer so ausgeführt, dass man den Infectionskeim an der gleichen Stelle und gleichzeitig dem schwangeren Thiere und dem nicht schwangeren, dem im Puerperium und dem im normalen Zustande einverleibte, und alle in der Folge unter den gleichen Bedingungen ernährte. Bei den Intoxicationen wurde darauf geachtet, zuerst die kleinste tödtliche Dosis zu bestimmen; so konnte man, nachdem der Werth bekannt war, anch wenn ein Controlthier fehlte, den Versuch ausführen, indem man das Gewicht und die beim Dosiren des Toxins bestimmte Giftigkeit im Verbältniss zu diesem bei Thieren derselben Gattung in Berechnung zog. Ebenso verfuhr man in einem Falle von Einimpfung eines schon öfters zum Experiment benutzten Mikroorganismus, damit ein einziges Controlthier für zwei Experimente bei schwangeren verwendet werden konnte.

Die als Infectionsstoffe verwendeten Mikroorganismen waren: B. coli (aus den weiblichen Geschlechtstheilen), B. diphtheriae in zwei verschiedenen Exemplaren), B. proteus mirabilis (aus Eihautresten erhalten), B. tuberculosis, pyocyaneus, pneumococcus, streptococeus pyogenes (in zwei Exemplaren, eines aus Eihautresten, eines von Angina diphtherica), Staphylococcus pyogenes aureus und zuletzt für Mischinfectionen. Streptococcus and Staphylococeus zusammen, ebenso B. diphthoriae und Streptococeus. 
Man versuchte auch den Typhusbacillus an zwei Thieren. Aber es gelang nicht, ihm die für die vorliegenden Versuche nöthige Virulenz zu verschaffen, und musste man darauf verzichten.

Jeder Mikroorganismus wurde vor dem Versuche durch fortgesetzte Ueberimpfungen namentlich im Peritoneum verstärkt. Vor jedem Experiment' wurde die Virulenz festgestellt, indem man die kleinste tödtliche Gabe bestimmte, um möglichst wenig zu irren, wenn man eine acute oder chronische Infection hervorrufen wollte.

$\mathrm{Zu}$ den Impfungen wurden jedesmal Culturen aus frischer Bouillon von 24 oder 36 Stunden verwendet oder solche aus menschlichem oder Rinderserum vermischt mit Bouillon, je nachdem sich der Mikroorganismus in der einen oder anderen Flüssigkejt besser entwickelte.

Es wurde immer daraul geachtet, schwangere Thiere an einer den Genitalien möglichst entfernten Körpergegend zu impfen, um Druck und Verletzungen des Fötus zu vermeiden. So wählte man für den subcutanen Weg den Rücken, für den endovenösen die Ohren. Nur zweimal, bei den Versuchen mit emulgirten Tuberkelbacillen in grösserer Menge, um eine acute oder subacute Infection hervorzurufen, war es nöthig, die Injection in die Peritonealhöhle zu machen.

Wenn der Tod erfolgte, wurde die Section sobald als möglich ausgeführt mit bacteriologischer Nachforschung. Fand man ein sterbendes Thier vor, so wurde es secirt, nachdem der Tod beschleunigt worden war. Zuerst warde die Bauchhöhle mit aller Vorsicht eröffnet, um das Schwangerschaftsproduct nicht zu verunreinigen. Der ganze Genitalschlauch wurde darauf nach gewissenhafter Unterbindung der zur Gebärmutter führenden Gefässe sorgfältig herausgenommen, so dass er nirgends mit dem Blute oder der Gewebsflüssigkeit in Berührung kam; das Ganze wurde dann in grossen, sterilisirten Petri'schen Flaschen aufbewahrt und sogleich bacteriologisch untersucht.

Der Einschnitt wurde mit sterilisirten Instrumenten gemacht, mit der bereit gehaltenen Platinöse wurde von der amniotischen Flüssigkeit entnommen und Aussaaten auf Platten und in Röhrchen mit verschiedenen Nährboden gemacht. Nach Freilegung wurde die Placenta in der Vitte gespalten und aus dem Centrum und von den Rändern wurde soviel Material als möglich zur Aussaat entnommen. In manchen Fällen wurde auch Blut aus der Umbilicalvene gesammelt. Zuletzt wurde die Haut in der Medianlinie 
eingeschnittan, die Leber freigelegt und wie die Placenta gespalten und mit dem Blute und zerstückeltem Gewebe wurden zahlreiche Culturen gemacht, auch mit dem Blute aus dem Herzen wurden Aussaaten gemacht. Als zweites Moment wurden aus dem gleichen Nateriale bacterioskopische Prïparate angefertigt, um sie mit dem Ergebniss der Calturen zu vergleichen und um den der Mutter einverleibten Mikroorganismus zu constatiren, der manchmal aber nur durch die Züchtung nachgewiesen werden konnte.

Man vollendete dann die Autopsie der Mutter, indem man aus den Exsudaten, den angetroffenen Ergüssen, dem Blute, den Organen bacterioskopische Präparate herstellte. Der durch die Reincultur erhaltene eingeimpfte Mikroorganismus diente später, dadurch verstärkt zu neuen Experimenten. Ganz das Gleiche wurde mit den Controllthieren (nicht schwangeren und noch nicht im Puerperium befindlichen) vorgenommen und dies Ergebniss mit mit dem vorhergehenden geprüft.

Wie aus der beigefügten Tabelle zu erseben ist, experimentirten wir zur Untersuchung der Jnfectionen mit 15 schwangeren Kaninchen und mit 15 schwangeren Meersehweinchen, mit 15 Kaninchen im Puerperium und 4 solchen Meerschweinchen. Als Controllthiere verwendeten wir bei diesen Versuchen 15 nicht schwangere Kaninchen und 15 ebensolche Meerschweinchen zum Vergleich mit den schwangeren Kaninchen und Meerschweinchen; zwei bezw. drei nicht schwangere und nicht im Puerperium befindliche Kaninchen und Meersehweinchen zum Vergleich mit den inficirten Thieren. im Puerperalstadium.

$\mathrm{Zu}$ Intoxicationen wählten wir zuerst das wohlbekannte 'l'oxin der Diphtherie in zwei Arten: eine schwächere aus gewöhnlicher alkalischer Bouillon, die andere, stärkere, aus Martin'scher Bouillon. Die erstere vom Werthe von $1 / 10 \mathrm{ccm}$ letaler Minimaldosis für ein Meerschweinchen von $500 \mathrm{~g}$, die andere von $1 / 20 \mathrm{ccm}$. Mit dieser wurde bei 5 sehwangeren Meersehweinchen experimentirt und bei 4 zur Controlle. In zweiter Linie verwendeten wir das Tuberkulin, wie es im serotherapeutischen Institut des Prof. Maragliano bereitet wird, vom Werthe von 0,70 für $100 \mathrm{~g}$ Meerschweinchen als tödtliche Dosis und von 0,60 (4 schwangere Meerschweinchen und 3 nichtschwangere zum Vergleich).

In der beigefügten Tabelle sind die Resultate unserer Untersuchungen zusammengestellt. Ohne uns über Einzelheiten der Versuche zu verbreiten, wollen wir nur erwähnen, dass die Einimpfungen 
bei schwangeren Kaninchen endovenös und subcutan und einmal intraperitoneal vorgenommen wurden; bei Thieren im Puerperium wurde abwechselnd intraperitoneal und subcutan und in 3 Fällen endovaginal und endouterin mit den obenerwähnten Organismen experimentirt.

Intection bei Thieren im Puerperium a p peritonealem a d suboutanem Wege.

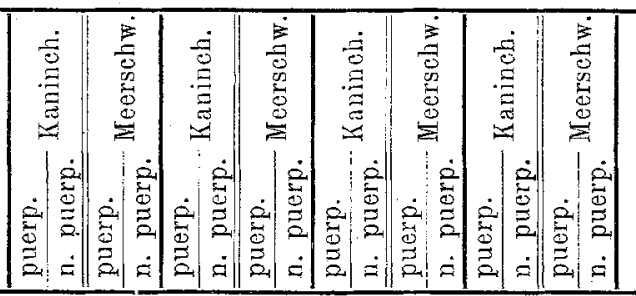

Staph. p. aur.

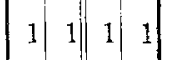

$1|1|$

Prot. mirabilis

. 1 (1) $111-$

$1----$

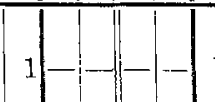

Es wurde der Uebergang in die Milch von zwei Mikroorganismen beobachtet, 1. Staphyl. in die Bauchhöhle injic., 2. Proteus mirabilis in das Rückenzellgewebe injic. Man fand den in das Peritoneum eingebrachten Mikroorganismus nicht.

Streptococc.

Virul. Streptoc.

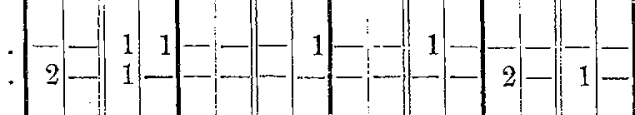

Auf vagin. und uterinem Wege.

Bei unverletzter Sohleimbaut gar keine Reaction, bei einem Versuch mit sebr virulenten Streptokokken wird das gesunde Thier getödtet, am herausgenommenen Uterus und Vagina findet man in Culturen und in Präparaten die Bakterien vollständig

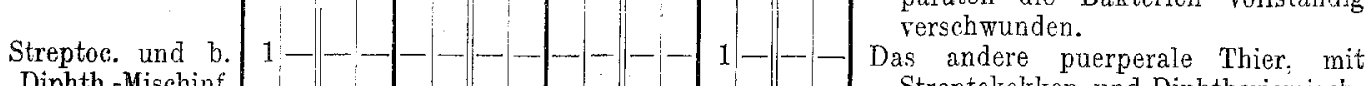
Diphth.-Mischinf. Streptokokken und Diphtheriemisehinfection behandelt, zeigte gar lseine Reaction und lebte noch 20 Tage nach dem Versuch.

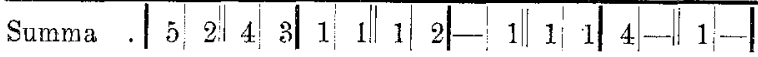

Nicht alle Versuche konnten im vorgeschrittenen Zustande der Schwangerschaft unternommen werden. Man versuchte schon bei ganz beginnender Schwangerschaft wegen der Schwierigkeit oder beinahe Unmöglichkeit beim lebenden Thiere, namentlich bei Kaninchen, den Zustand zu beurtheilen. Einige Fälle ron eben beginnender Schwangersehaft wurden ausgeschlossen. Obwohl unsere Versuche noch fortgesetzt werden, um das Material für eine vollständigere Beurtheilung zu vermehren, ziehen wir in dieser vorläufigen Mittheilung dennoch die Schlüsse, die aus dem Ergebniss unserer Experimente folgen und stellen sie in der oben angeführten Tabelle 
zusammen. Diese Schlüsse halten wir für wichtig, hauptsächlich bei schwangeren Thieren, mit denen auch mehr experimentirt wurde.

Als erste Thatsache fällt aul die weit grössere Anzahl acuter Infectionen bei schwangeren Thieren als bei nicht schwangeren; dann die weit grössere Zahl von tödtlichem Ausgang bei schwangeren Thieren, was beweisen würde, dass bei letzteren eine grössere Disposition zu Septikämien und eine geringere Widerstandskraft des Organismus gegen Infectionen besteht.

Diese Beobachtung war um so aufallender, je weiter die Schwangerschaft vorgeschritten war; in jenen Thieren, bei denen die Schwangerschaft eben begonnen hatte, fand man keinen Unterschied von den normalen Controlthieren.

Bei der Section dieser schwangeren Thiere sah man eine grosse Infiltration von eingespritzten Mikroorganismen im Parenchym der Organe und im circulirenden Blute. So für den Diphtheriebacillus, den man sonst selten im letzteren nachweisen kann, constatirte man es einmal in 4 Fällen. Es ist bekannt, dass man sonst diesen Bacillus beinahe bei allen Thierexperimenten nur an der Injectionsstelle localisirt findet. Ein Beweis, wie der Durchgang der unter die Haut gebrachten pathogenen Mikroorganismen ins Blut und in die Organe erleichtert und in den Organen die Disposition zur Infiltration erhöht sei, begleitet vielleicht auch von einem geringeren Ausscheidungsvermögen wegen des Kreislaufshindernisses hauptsächlich bei vorgeschrittener Schwangerschait. Deshalb erfolgt auch die grösste Zahl der Todesfälle wegen acuter Infection oder Septikämie, - aus der Tabelle ersichtlich - was auch schon bei Lebzeiten durch die starke Reaction gegen Infectionen sich zeigt, die viel stärker bei schwangeren Thieren ist, als bei nicht schwangeren und durch die viel höheren Temperaturen bei schwangeren Thieren im Vergleich zu den Nichtschwangeren mit Unterschieden von $1^{0}$ bis $1.5^{\circ}$. In Bezng auf Abort oder bebeginnenden Abort mit Riss des Fruchtsackes beobachtete man, dass ersterer bei Kaninchen niemals vorgekommen ist; nur einmal beobachtete man den einfachen Riss des Fruchtsackes in Folge acuter Infection mit B. coli. Es ist jedoch zu bemerken, dass die Zahl der hochschwangeren Kaninchen, mit denen experimentirt werden konnte, eine sehr geringe war.

Bei Meerschweinchen fand man 2 mal den einfachen Riss des 
Fruchtsackes, einmal nach Infection mit B. coli, ein andermal nach B. diphtheriae. 2 mal beobachtete man vollständigen Abort, einmal nach Einimpfung von Streptokokken, ein andermal nach B. diphtheriae und noch einmal, wie wir weiter unten erwähnen werden, durch das Toxin dieses letzteren.

Im Gegensatz zu dem, was man bei der Infection mit allen anderen Mikroorganismen constatirte, sah man bei Infectionen durch B. diphtheriae bei schwangeren Meerschweinchen eine grössere Widerstandskraft und zwar (s. Tabelle) starben von 4 sehwangeren und 4 nicht schwangeren geimpften Controlthieren nur 2 schwangere, aber alle nicht schwangeren, und man konnte bei der Section eines der ersteren das seltene Factum beobachten, dass ans dem in grosser Menge ausgesäten Herzblute nur ganz vereinzelte Colonien des B. diphtheriae aufgingen.

Was die Constatirung des Mikroorganismus in der Placenta, in der amniotischen Flüssigkeit und was mehr interessirt im Blute und in den Organen des Fötus betrifft, hatten wir folgende Ergebnisse: Positiv war das Ergebniss in 10 Placenten von Kaninchen, in 8 von Meerschweinchen; in 3 amniotischen Flüssigkeiten ron Kaninchen, in 2 von Meerschweinchen; 4 mal fand man ihn im Blut und in den Eingeweiden von Kaninchenföten, 3 mal in solchen von Meerschweinchen. Es ist zu bemerken, dass die Injectionen bei Kaninchen zum Theil endovenös, zum Theil subcutan gemacht worden waren, bei schwangeren Meerschweinchen immer (ausgenommen für den Tuberkelbacillus) in das Zellgewebe und manchmal in hoher Dosis.

In der Placenta fand man oft eine grosse Menge von Mikroorganismen und manchmal mikroskopisehe Veränderungen wie Erweichungen.

In der amniotischen Flïssigkeit: Differenz der Färbung, Blutergüsse, mehr oder weniger ausgesprochene Dichtigkeit, geringere oder grössere Menge, blutiges Serum in einem Falle von Infection mit B. coli von rothbrauner Farbe, mehr ausgesprochen, in einem Falle von Infection durch B. pyocyaneus und streptococcus; dichter und beinahe gelatinös bei Infection durch B. diphtheriae wie auch durch das Toxin desselben.

Die Föten fand man macerirt oder in beginnender Maceration in Folge Infection mit Streptokokken, B. pyocyaneus und B. coli, alles Vikroorganismen, welche gleich in den ersten Tagen nach der Injection die höchsten beobachteten Temperaturen erzeugten und 
vielleicht dadurch schon ron Anfang an das Absterben des Fötus verursachten.

Der Anwesenheit des der Mutter injicirten Mikroorganismus im Blute oder in den Eingeweiden des Fötus wurde sorgfältig nachgeforscht wcgen der grossen Wichtigkeit, den in der Pathologie der Schwangerschaft der Durehgang der Infectionskeime von Mutter auf Kind einnimmt, und mehr noch wegen der Bedeutung. für die geburtshülfliche Praxis.

Viele Autoren streiten sich noch darüber, ob beim Weibe ein solcher Durchgang stattfinden kann; Eppinger und Morisani sahen ihn nicht für den Milzbrandbacillus, ein Bacillus der am häufigsten das Blut und alle Organe überschwemmt. Andere fanden ihn, so D. Vitanza für die Cholera; Braul, Davaine, Bollinger erkennen für die Placenta die Eigenschaft eines vollkommenen Filters an, das im Stande ist, nicht einmal den Milzbrandbacillus durchzulassen. Endlich giebt eine grosse Zahl Uebergang von Mutter auf Kind zu im Falle von Verletzung der Placenta, schliessen ihn aber aus bei intacter Placenta. Auf Grund der bei unseren Experimenten gemachten Beobachtungen und aus dem Ergebniss derselben stimmen wir der Ansicht dieser letzteren bei, d. h. der Uebergang von Mutter auf Fötus ist nur dann möglich, wenn die Placenta verletzt ist und dies wegen zwei constatirter Thatsachen: erstens weil in den Fällen von positivem Befund von der Mutter eingeimpften Mikroorganismen in den Föten man die Placenta in der Mehrzahl der Fälle erweicht fand, zweitens wegen der geringen Zahl von positiven Befunden im Vergleich zu den positiven Befunden in der Placenta (10 auf 15 geimplte Kaninchen, 8 auf 15 Meerschweinchen, im Blute und den Lingeweiden der Föten 4 bei den Kaninchen, 3 bei den Meersehweinchen). Weiter wurde festgestellt, dass beinahe in allen Fällen von positivem Befund die Autopsie erst verschiedene Stunden nach dem Tode vorgenommen

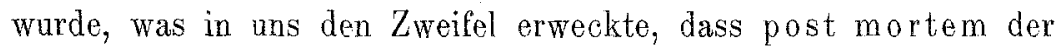
Uebergang anch durch die unverletzte Placenta möglich werde, wie es für den Streptococcus bei Erysipel geschieht, bei dem es selten ist, den Coccus bei Lebzeiten im Blute des Kranken zu finden, während man es nicht selten beim Leichnam trifft.

Nach dem Tode, wenn die Gewebe ihre Widerstandskraft und die Placenta ihre Eigenschaft als Filter verloren, oder auch in der Agonie kann der Uebergang des Infectionsstoffes, der vorher in der Placenta zurückgehalten worden war, ron Mutter auf Fötus vor 
sich gehen. Ein Beweis, der uns in dieser Ansicht bestärkt, ist, dass wir für denselben Mikroorganismus in einem Falle, als die Section bei noch lebender Frucht gemacht und sofort der Genitalschlauch entfernt wurde, der Befund negativ war, während er in einem anderen Falle, wo erst mehrere Stunden nach dem Tode secirt wurde, der Befund positiv war. So könnte es auch beim schwangeren Weibe geschehen, dass bei einem Theil der Fälle mit positivem Befunde von Infectionskeimen im Fötus übergegangen aus der Mutter, dieser Uebergang post mortem oder in der Agonie geschehen sei und bei Lebzeiten noch nicht vorbanden war. Diese interessante Frage bedarf noch einer genaueren Prüfung mit Hülfe einer grösseren Reihe von Thierexperimenten; erst dann könnte man ein bestimmteres Urtheil abgeben, als es jetzt beim heutigen Stande unserer Versuche möglich ist.

Was die mit Injectionen von Toxizen bei Schwangeren angestellten Experimente angeht, so fand man für die Diphtherie, dass von fünf mit der vorher bestimmten minimalen tödtlichen Dosis injicirten schwangeren Meerschweinchen drei das Experiment überlebten, während von vier gleichzeitig inoculirten nichtschwangeren nur eins davonkam.

Einmal trat vollständiger Abort ein, ein anderes Mal blieb es beim Riss des Fruchtsackes stehen, und dies bei nicht weit vorgeschrittener Schwangerschaft. Von den von Meerschweinchen ausgestossenen Föten war einer vor der Reife abgestorben, der andere, lebend, starb gleich, während die Mutter keine Milchsecretion zeigte. Die gleiche Thatsache des Fehlens der Milch zeigte sich bei einem Meerschweinchen, das zwei lebendige Föten gebar nach der Injection des B. diphtheriae; diese starben wegen Mangel an Nahrung.

Bei den durch das Toxin gestorbenen Meerschweinchen sowie bei denen, die durch Einimpfung des B. diphtheriae zu Grunde gingen, fand man eine auffallende Dichtigkeit der amniotischen Flüssigkeit (beinahe gelatinös). Die locale Reaction ist sowohl bei den mit Toxin als bei den mit B. diphtheriae geimpften Meerschweinchen weniger stark bei den schwangeren als bei den nicht schwangeren. Bei den mit B. diphtheriae inficirten, wie auch bei den mit Toxin inoculirten geborenen Föten findet man eine grosse Schwäche der Entwiekelung; bei den Jetzteren findet man etwas pleuralc und peritoneale Flüssigkeit, vielleicht ein Zeichen des Uebergangs des toxischen Prineips. Doch kann man den Tod nicht 
allein diesen Veränderungen zuschreiben, wohl aber dem Mangel der Milch bei der Mutter; es könnte sich aber um eine durch das Toxin hervorgerufene Frühgeburt handeln oder um Unterdrückung der schon begonnenen Milchsecretion.

Das Tuberkulin, mit welchem ebenfalls experimentirt wurde, ergab nicht die Resultate wie bei der Diphtherie. Von vier mit der minimal tödtlichen Dosis geimpften schwangeren Meerschweinchen sind zwei gestorben, zwei blieben am Leben. Von den drei nicht schwangeren Controllthieren sind zwei gestorben, eins kam davon. Dies Ergebniss ist zu unbestimmt, um ein Urtheil über

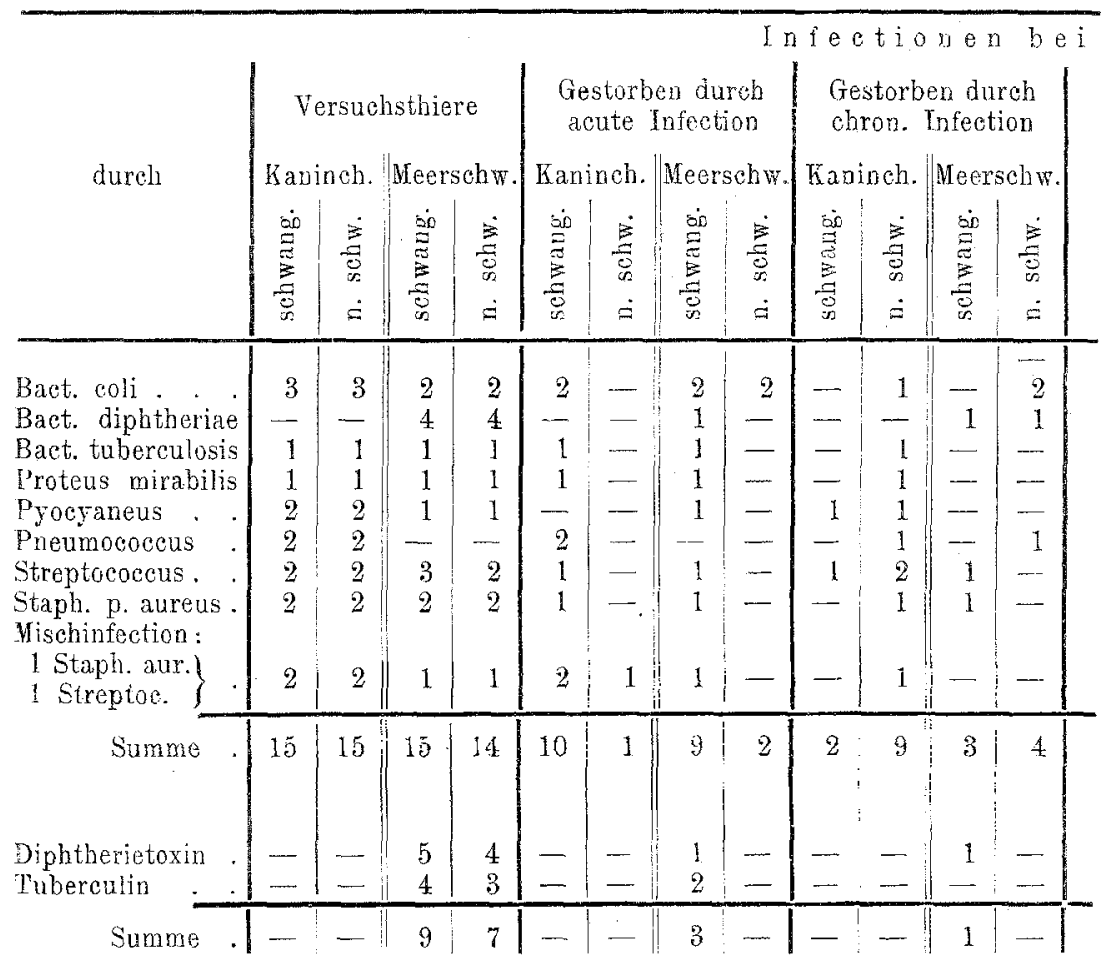

die grössere oder geringere Toleranz der schwangeren Thiere für dieses tuberkulöse Gift abrugeben, welches aber keinen Abort herbeiführte. Die Experimente sind in viel zu geringer Zahl angestellt, um ein Toxin wie die Diphtherie mit einem Protein wie das Tuberkulin in ihrer giftigen Wirkung auf schwangere Thiere zu vergleichen.

Die entzündliche Reaction an der Injectionsstelle des Tuberkulins scheint bei den schwangeren Meerschweinchen geringer als 
bei den nichtschwangeren; im Uebrigen sind die Veränderungen jdentisch, man sah auch keine bedeutende makroskopische Modifcation in dem Schwangerschaftsproduct nach der Tuberkulininjection ausser in einem Falle die dunkelrothe Färbung der amniotischen Flüssigkeit wegen Blutergusses und die Congestion in der Leber des Fötus, begleitet von einem gleichen in der Placenta.

Die fünf Versuche bei Kaninchen im Puerperium und die vier bei Meerschweinchen im Puerperium wurden zum Theil durch subcutane und endovenöse Injection vorgenommen, zum Theil durch endovaginale und uterine Injection.

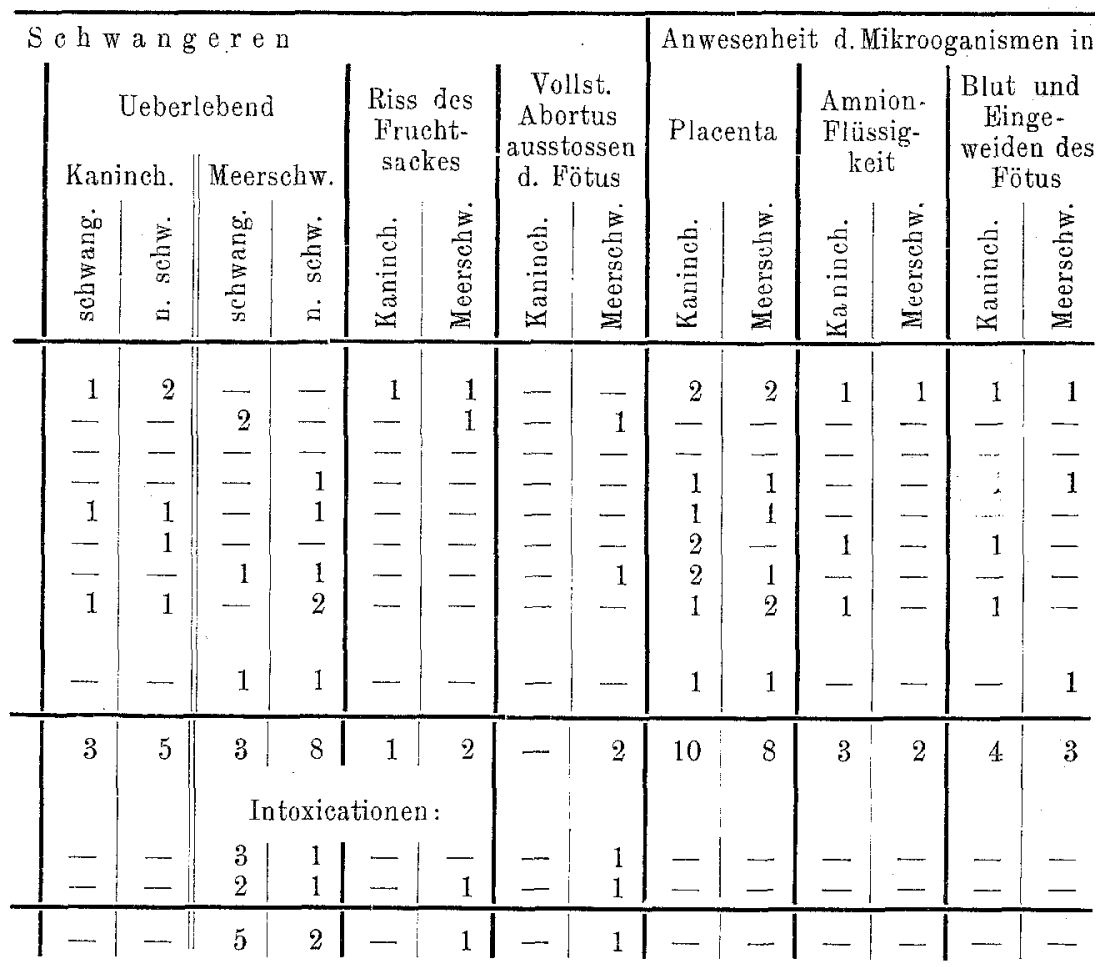

Mit dem Staphylococcus pyogenes aureus wurde je ein Kaninchen und ein Meerschweinchen, welche beide geworfen hatten, zu gleicher Zeit mit den nicht im Puerperium befindlichen Controllthieren geimptt; alle in die Peritonealhöhle. Dieser Mikroorganismus befand sich in hoher Virulenz. Dieselbe war schon bei Beginn ziemlich hoch und wurde durch mehrfache Durchgänge gesteigert, da er von Angina scarlatinosa stammte. Das Ergebniss war das gleiche letale beim Kaninchen, mochte es sich im Puer- 
perium oder ausserhalb desselben befinden; von den Heerschweinchen blieb nur das im Puerperium befindliche am Leben.

Bei diesen beiden Thieren konnte man in der sowohl bei Lebzeiten als im Tode aseptisch entnommenen Milch die Anwesenheit des injicirten Mikroorganismus constatiren. Auch Karlinski hat den Staph. pyog. aur. in der Milch einer an puerperaler Pyämie erkrankten Fran nachgewiesen. Bei dem überlebenden Meerschweinchen hörte die Milchsecretion nicht auf, sondern erhielt sich noch für verschiedene Tage.

Mit Proteus mirabilis, der aus Eiresiduen stammte und der zu verschiedenen Proben durch den thierischen Organismus durchgegangen war, injicirte man subcutan in ziemlich grosser Dosis ein Kaninchen und ein im Puerperium befindliches Meerschweinchen, sowie zwei Thiere der gleichen Gattung, die nicht im Puerperium waren. Exitus letalis für das nicht puerperale Kaninchen und für beide Meerschweinchen: es kam nur das puerpere Kaninchen davon. Bei diesem fand man auch durch Cultur die Anwesenheit des injicirten Protens mirabilis in der Milch, die Secretion dauerte jedoch nicht lange; am zweiten Tage nach der Injection warde die Milch spärlich, sie tritt aus den Warzen nur durch starkes Pressen aus und in der Form von kleinen Flöckchen; das verwendete Kaninchen hatte vor der Infection einen richtigen Milchstrahl bei Druck auf das Euter.

Der dritte Mikroorganismus konnte nur bei einem puerperen und bei einem nicht puerperen Meerschweinchen geprüft werden. Die Injection wurde mit dem Streptococeus in die Peritonealhöhle gemacht; wegen der grossen Virulenz, obwohl in schwacher Dosis injicirt, verursachte sie den Tod beider Thiere; beim puerperen in 8 Tagen, beim nicht puerperen in 48 Stunden. Beim ersteren konnte man trotz wiederholter Versuche die Anwesenheit des Streptococeus in der Milch nicht nachweisen.

Aus dem vorliegenden Resultat der Infectionen bei Thieren, die geworfen hatten, im Vergleich mit anderen Thieren der gleichen Gattung, die nicht im Puerperium standen - in zu geringer Zahl ausgeführt - scheint hervorzugehen, dass kein Unterschied in Betreff der grösseren oder geringeren Widerstandskraft gegen infectiöse Processe bestehe zwischen Thieren im Zustande des Puerperiums und solchen nicht in diesem Zustande.

Vielmehr soheint die Thatsache von einiger Wichtigkeit zu sein, dass ein Durehgang der unter die Haut und in's Peritoneum der 
Wöchnerin eingespritzten Mikroorganismen in die Milch besteht, was eine Erklärung abgeben könnte, für die Uebertragung von infectiösen Processen durch die Amme auf den Säugling und uns ermahnt, in zweifelhaften Fällen die bakteriologische Untersuchung der Milch nicht zu unterlassen.

Die letaten Versuche betrafen die Möglichkeit mit virulenten Organismen auf endovaginalem oder uterinem Wege Thiere, die vor Kurzem geboren haben, zu inficiren, ohne die Schleimhaut zu verletzen. $\mathrm{Zu}$ diesem Zwecke verwendeten wir zwei puerpere Kaninchen und zwei Meerschweinchen, wir wählten zwei der giftigsten Mikroorganismen, den schon in den vorhergehenden Versuchen angewendeten Streptococcus noch verstärkt und einen Diphtheriebacillus, der Meerschweinchen von $350 \mathrm{~g}$ in der Dosis von $1 / 2 \mathrm{ccm}$ eingespritzt in 2 oder 3 Tagen tödtete. Die Injection wurde folgendermaassen gemacht. Das Thier wurde auf den Operationstisch festgebunden mit den Hinterbeinen nach oben, eine kleine Hohlsonde führte man in die Vagina ein und suchte sie in die Uterushöhle vorzuschieben. Wenn man dies erreicht zu haben glaubte, setzte man eine Spritze an von 1,5 ccm Culturinhalt und spritzte diesen plötzlich mit Gewalt in die Sonde. Gleich nach Beendigung dieser Operation wurde die Sonde rasch herausgezogen und die Vagina vollständig durch einen comprimirten Wattetampon verschlossen. Auf diese Weise wurden zwei Kaninchen und ein Meerschweinchen im Puerperalstadium injicirt. Am Morgen des folgenden Tages constatirte man, dass der Tampon ausgestossen war, und man fand eine einfache Hyperämie der Vagina, ohne irgend welche heftigere Reaction. Am zweiten Tage tödtete man eines der Kaninchenweibchen und nach Secirung der herausgenommenen Vagina und Uterus fand man keine Spur von Verletzungen oder krankhaften Veränderungen der Schleimhaut weder der Vagina noch des Uterus. Mit der Platinöse schabte man die Schleimhaut an verschiedenen Stellen ab und mit dem gewonnenen Materiale impfte man verschiedene Bouillon- und Agar-Röhrchen, ebenso wurden bakterioskopische Präparate hergestellt. Durch diese wie anch durch die Culturen konnte man den eingespritzten Streptococcus nicht nachweisen, der vollständig versehwunden war; auch beobachtete man in der Folge in den anderen beiden injicirten Thieren keine Spur von Infection. Das letzte Thier im Puerperalstadium mit B. diphtheriae und Streptococeus in Mischcultur injicirte zeigte auch gar keine Erscheinungen und lebt jetzt noch, nach dem 
20 Tage seit der Einbringung des Virus verflossen sind. Diese Ergebnisse könnten beweisen, dass die Einführung selbst der stärkstgiftigen Mikroorganismen auf vaginalem oder uterinem Wege nicht im Stande sei, bei unverletzter Schleimhaut im Kaninchen oder Meerschweinchen irgend eine Infection hervorzurufen.

Es bleibt zu untersuchen, ob es möglich sei, dies zu erreichen, indem man durch leichtes Ritzen der Schleimhaut kleine Continuitätstrennungen hervorbringt durch Abschabung des Epithels. Jedenfalls sind mehr Versuche nöthig, über diese Thatsache definitiv zu urtheilen.

Unterdessen kann man aus den oben beschriebenen an 79 Thieren ausgeführten Versuchen, die in der angeführten Tafel zusammengestellt sind, bei deren Prüfung ich von Dr. Gioielli bestens unterstützt wurde, vor der Hand folgende Schlüsse ziehen. Ich sage vor der Hand, denn sie müssen durch eine grössere Versuchsreihe erst bestätigt werden.

1. Durch Injiciren nach der oben angeführten Art und den angegebenen Bedingungen bei 30 Kaninchen und 29 Meerschweinchen in schwangerem und in nicht schwangerem Zustande zur Controlle von B. coli, proteus mirabilis, B. diphtheriae, B. tuberculosis, pyocyaneus, pneumococeus, streptococeus pyogenes, staphylococeus pyogenes aureus. Für Mischeulturen von Streptococcus mit Staphylococeus, von B.diphtheriae mit Streptococcus erhält man ceteris paribus vor Allem eino weit grössere Zahl von acuten Erkrankungen bei schwangeren Thieren als bei nicht schwangeren; die Zahl der tödtlichen Ausgänge ist ebenfalls weit grösser für schwangere als nicht schwangere, die mit den gleichen pathogenen Keimen unter gleichen Bedingungen inficirt worden sind.

2. Der oben angeführte Unterschied in der grösseren Leichtigkeit acute Phänomene und Tod bei Schwangeren hervorzurufen, war um so deutlicher und grösser, je weiter die Schwangerschaft rorgesehritten war, so dass man in Fällen von beginnender Schwangerschaft keinen Untersehied von den nicht schwangeren Controllthieren wahrnehmen konnte.

3. Wie für die anderen Erscheinungen in Folge experimenteller Infectionen, stellte man für die Temperatur bei den Schwangeren ein Mittel von $1,5^{\circ} \mathrm{C}$. höherer Temperatur fest als bei den nichtschwangeren Controllthieren. 
4. In Folge ron experimentellen Infectionen waren Aborte sehr häufig.

Dem oben Angeführten entgegengesetzte Ergebnisse wurden für den B. diphtheriae beobachtet, und zwar fand man eine grössere Widerstandskraft gegen seine Wirkung bei schwangeren Thieren als bei nicht schwangeren.

5. In einer verhältnissmässig hohen Zahl von Fällen fand man in der Placenta (bei zehn Kaninchen und drei Meerschweinchen) und im Blute der Föten (vier Kaninchen und drei Meerschweinchen) die $\Lambda$ nwesenheit des injicirten pathogenen Keimes.

6. Arch gegen das Diphtherietoxin fand man bei schwangeren grössere Widerstandskraft als bei nichtschwangeren Controllthieren; für das Tuberkulin war das Ergebniss unbestimmt.

7. Für die experimentelle Infection von Thieren im Puerperium konnte man bis jetzt keine bemerkenswerthen Verschiedenheiten beobachten im Vergleich zu den nicht im Puerperium befindlichen Thieren.

8. Der Durchgang der den Wöchnerinnen eingespritzten Mikroorganismen in die Milch scheint ersehöpfend bestätigt zu sein.

Die wichtigsten Ergebnisse zur Lösung unserer hentigen Frage über die Indication zur Unterbrechung der Schwangerschaft wegen innerer Krankheiten, wegen derer ich eine grössere Zahl von Experimenten ausführte in der Erwartung, dass sie noch weitere Bestätigung erfahren werden, sind jene, welche die intensivere Phenomenologie hervorgerufen durch die pathogenen Keime, ihre grössere und raschere Ausbreitung (ausgenommen allein der B. diphtheriae) mit tödtlichem Ausgange bei schwangeren Thieren und ibre geringere Widerstandskralt gegen die Tnfection bestätigen. Selrr beachtenswerth ist die Thatsache, dass, je weiter vorgeschritten die Schwangerschaft ist, desto schneller, stärker und heftiger die Invasion und desto geringer die Widerstandskraft ist.

Klar ist die Folgerung, dass in Fällen von Schwangerschaft. complicirt mit einer Infectionskrankheit auch wissenschaftlich die künstliche Unterbrechung derselben angezeigt ist und diese Unterbrechung muss so früh als möglich vorgenommen werden, da man weiss, dass je weiter die Schwangerschaft fortschreitet, um so grösser wird die Wucht und die Schnelligkeit der Invasion, um so geringer die organische Widerstandskraft und deshalb grösser die Gefabr für die Mutter. 
Solite die weitere durch die Experimente erwiesene Thatsache sich bestätigen, dass die Intensität der Infectionskeime nicht höher und die Widerstandskraft der Gewebe bei Thieren im Puerperalstadium nicht geringer ist, so würden wir noch viel mehr ermuthigt, die oben angeführte Folgerung anzuwenden.

Die hauptsächlichste Erwägung, wegen derer die Kliniker bis heute zum grössten Theil verhindert wurden, dieser Indication die verdiente Aufmerksamkeit zu schenken, war die wegen des Verlustes des Sehwangerschaftsproductes.

Aber auch diese Erwägung würde, ich möchte sagen, beseitigt durch unsere Ergebnisse, die uns zeigen, dass die Aborte in Folge von Infection des mütterlichen Organismus sehr häufig sind, häufig auch der Uebergang des Infectionsstoffes auf den Fötus und seine Anhänge. Ich sagte bis heute, weil mir wirklich scheint, wenn ich die medicinische Literatur durchgehe, dass die ersten deutlichen Erklärungen der Opportunität nicht nur, sondern der Nothwendigkeit der Unterbrechung der Schwangerschaft wegen Infectionskrankheiten in der Arbeit von Martin (1891) niedergelegt seien, von Maragliano (1893) und ron mir (1890) hauptsächlich in Betreff der Wirkung von Tuberculose auf Schwangerschaft.

In dieser Hinsicht habe ich heute nichts $\mathrm{zu}$ streichen und nichts zuzufügen zu dem, was ich 1893 in einer meiner Abhandlung geschrieben habe [Ueber die geburtshülfliche Prophylaxis in der Wohnung $\left.{ }^{1}\right)$, und was jch hier wörtlich anführe. "Es ist meine Ueberzengung, die ich schon bei manch anderer Gelegenheit ausgesprochen habe, dass die Indicationen für die Unterbrechung der Schwangerschaft wegen jnnerer Krankheiten noch nicht genügend erforscht und gewertet sind. Das Zuwarten, wenn auch manchmal leichter und weniger verantwortlich für den Geburtshelfer, setzt uns doch häufig unerwarteten Katastrophen aus. Es ist, meine ich, Pflicht des Arztes individuell in den einzelnen Fällen von Tuberculose die Widerstandskraft des Kranken zu schützen, und wenn wir im Zweifel sind, ob sie noch die Beschwerden der anderen Schwangersehaftsmonate und der Geburt aushalten könne, ist es auch seine Pflicht, die Schwangerschaft zu unterbrechen, in welchem Stadium sie sich auch befinde.

1) Bossi, Sulla Profilassi ostetrica a domicilio. Archivio di Ost. e yinec. Anno 1. Faso. 4, 5, 6, 7. Napoli. 


\section{Literaturverzeichniss.}

Ueber den Durchgang der Infectionskeime von der Mutter auf Fötus haben wir in der. Literatur viele Arbeiten und verschiedene Meinungen. Beginnend mit den aller virulentesten dem Milzbrand, für den man die meisten Beobachtungen hat, finden wir Autoren, die ihn als möglich erwiesen, ehronologisch geordnet.

\section{Milzbrand.}

1) Perroncito Arbeit im Rendiconto della R. Accademia Medica di Torino. 1882. p. 930. - 2) Diesem folgen 2 Arbeiten von Sangalli, Bacterii del Carbonchio in feto di giovenca. Rendiconto R. Istituto Lombardo di Scienze 1882. Vol. 15. p. 668. Griffini, Sull' Immunità contro it carbonchio. R. Istituto lombardo di Scienze Anno.1882. Vol. 15. p. 526. -- 3) Strauss, Transmission de quelque maladie virnlente en particulier du charbon de la mère an foetus. Archiv de physiologie. 1883. - 4) Birch-Hirschfeld, Revne des sciences médicales. 1885. Tom. 26. p.46. - 5) Paltauf und Marchand, Dieser letztere glaubt, dass der Durchgang während der Ablösung der Placenta wegen Verletzung der Zotten geschehen liönne. - Virchow, der den Durchgang als möglich zugiebt bei Veränderungen der Placenta. 7) Massa, Studi bacterjologici solla trassmissione del bacillus Anthracis. Riforma medica. 1896. No. 28. - 8) De Para, Werı die Placenta nicht immer für die Bacillen des Milzbrandes durchgängig ist, so ist sie es immer für die toxischen Stoffe. Atti società Ital. di Ost. e Ginecol. Congresso Napoli. 1900.

Unter den Autoren, welche diesen Durchgang des Milzbrandbacillus nicht fanden:

1) Brauel, Davaine, Bollinger. - 2) Morisani, Sopra un caso di pustula maligna non trasmessa da madre a feto. Morgagni 1886 . p. 523. - 3) Romano, Sopra un caso di pustula maligna non trasmessa da madre a figlio. Morgagni 1888. p. 438. - 4) Epping er 1886, Diese Autoren nehmen die Placenta als vollkommenen Filter an, welcher nicht durchbrochen werden kann.

\section{Typhus.}

Für die typhöse Infection fanden den Durchgang auf den Fötus an Frauen, die wegen Typhus abortirten:

1) Reher, Zur Aetiologie des Abdominaltyphus. Berliner klin. Wochenschrift. 1886. S. 389. - 2) Neuhaus, Weitere Untersuchungen über den Bacillus des Abdominaltyphus auf den Fötus. Berliner klin. Wochensebrift. 1885. S. 389. -- 3) Jannisensky, Uebertragung des Typhus auf den Fötus. Münchener med. Wochenschr. Bd. 38. 1893. - 4) Freund und Levy. 5) Etienne, La fièrre typh. du foetus, Arch. de Gyn. et Tocol. 1896. p. 509. - 6) Eberth, Geht der Typhusorgan. anf den Fötus. Fortschritte der Med. 1889. - 7) Hildebrand, Zur Kasuistik der Placenta. Uebergang der Typhusbacillen. Fortschritt der Med. 1889. No. 23. S. 889. - 8) Giglio, Ueber den Uebergang des Milroorg. d. Typhus. Centralblatt für Gy, 1890. S. 819. 
- Balp, Ln caso di tifo addom. Gazzetta medica Lombarda. 1891. p. 369. -. 10) Frascani, Osservazioni cliniche e ricerche sul passaggio sperimentale delle tifo. Rivista gener. di Clinica Ital. 1892. No. 12-16. - 11) Ernst. Negative Ergebnisse hatten.

1) Fraenkel u. Simmonds, Weitere Untersuchungen über die Aetiologie des Abdominaltyphus. Zeitschr. für Hygiene. 1887. S. 144. - 2) Resinelli, Note batteriologische su di un aborto per tifo. Annali di Ost e Ginec. 1895-1896. p. 694 .

\section{Cholera.}

Für den Cholerabacillus constatirte den Uebergang:

1) Tizzoni u. Cattani, Ueber die Uebertragung der Cholera inf. von der Mutter auf den Fötus. Centralbl. für die med. Wissenschaften. 1887. No. 8. S. 131. (Thierversuche.) - 2) Vitanza, Sulla transmissione dell' infezione coler. da madre a feto. Riforma medic. 1890. (Fand im menschlichen Fotus den Bacillus nicht, wohl aber durch Experiment beim Thier.)

\section{Pneumacoocus.}

Den Uebergang des Pneumococcus von Mutter auf Fötus haben beobachtet.

1) Pessari, Sul passaggio dell' infezione da pneumococco. Bolletin. dell R. Academie medica di Genova. 1886-1887. - 2) Foà e Bordoni-Uffreduzzi, Sull arione del pneumococco e del suo passaggio da madre a feto. Riforma medic. 1887. No. 39 .

\section{B. Morva.}

Zulect constatirten Pessari und Guarneri den Uebergang des Rotzbacillus, Sopra un caso di morva nell' nomo. Atti R. Academia medica di Roma am 13. 1886-87.

6. Tuberculose.

Maffucci, Der Uebergang des Tuberkelbacillus ist selten, häufiger der Durchgang des Giftes von Mutter auf Kind. Policlinico. 1894. p.33. 1. März.

Eebergang von Mikroorganismen in die Milch.

1) Escherich, Cohn, Neumann and Honigmann gaben zu, dass die pathogenen Keime in den Milchgängen gefunden werden können. - 2) Hehling, Trattato fisiol. e pat. puerper. nimmt die Ferbreitung beinahe nur auf lymphatischem Wege an, selten die der Blutbahn und von innen nur bei Thieren durch Injection in die Vena jugularis. - 3) Löhlein, Assistent von Fehling, fand in der Milch den Staphyloc. pyog. abl., seltener den aureus. -. 4) Princi giebt zu, dass die Frauenmilch auch bei gesunder unverletzter Brust pathogene Milroorganismen enthalten könne. In einem inficirten Organnismus, dessen Milroorganismen im Blute circuliren, können sie durch die Milchsecretion ausgeschieden werden, hier verfolgen die Bacillen, um zur Milch zu gelangen die Blatbahn. Arch. d. Ost. e Ginecol. 1899. p. 303. - 5) Calandra, H. b. piocianico prodigiosus, bact. coli, pneumobaccill. d. FriedJänder el $b$. della difterite del colera injettati sollo cate non passano nel latte Arch. di Ost. e Ginecol. 1899. p. 303. - 6) In Betreff des Staphylococcus pyogen. aureus hat Karlinsky seine Gegenwart in der Franenmilch constatirt bei eine an Puerperalinfection erkrankten, ebenso in Versuchen mit Kaninchen.

Ein experimentell. Beitrag zur Kenntniss des Pyocyan. des Neugeborenen.

De Bonis, Bei Darminfectionen der Kinder fand er in den Stühlen sehr reichlich den pyocyaneus aureus. VI. Congress Medicin. 1895. 